\title{
Virtue in now-antiquated textbooks
}

\author{
General relativity is not everybody's interest, but there is much to be said for rereading Tolman's monograph on \\ the subject, first published in 1935, not just for what it says but for what it fails to say.
}

OCCASIONALLY, most people find themselves forced to read through books and papers they have not looked at for decades, in a kind of instant refresher course in a forgotten subject. It is always a frustrating experience. The old textbooks have the advantage of being familiar, even to the extent that the meaning of the old diagrams stands out without help from the legends beneath them. On the other hand, the old books that have occupied shelfspace all these years have probably long since been superseded by much more effective ways of learning. It might even be discovered that the old books embody precisely those obscurities that made the subject difficult to understand in the first place - and which have made the refresher course necessary.

What follows is an account of an attempt to learn about general relativity in a hurry, almost exactly 50 years ago. The occasion was a series of seminars given at Oxford by the relativist E.A. Milne, whose claim on public attention was the doctrine of "kinematical relativity", which holds that the puzzling business of Lorentz transformation in special relativity tumbles out from a careful accounting for the times spent by light in travelling to and from distant moving objects. Milne had had a bad time in captivity during the Second World War, and had returned the worse for wear.

His seminars, on Tuesday and Friday afternoons in a room at the end of the first floor of the Clarendon Library, caught the attention of half a dozen people with exceedingly diverse interests. One of the most faithful was M.J.S. Dewar, a theoretical chemist who has spent most of his working life at Austin, Texas. Milne's style was Socratic. $\mathrm{He}$ enjoyed his seminars best when there was an argument. That tendency afterwards became apparent in Milne's public exchange of vitriolic correspondence with Herbert Dingle, which entertained a year's cohort of Nature readers in the early 1950 s.

Of necessity, textbooks on relativity were then few and far between. My college library had only two - Eddington's Relativity, Protons and Electrons and Tolman's Relativity, Thermodynamics and Cosmology, both published in the mid-1930s. Eddington's book is a startling read; among other things, it calculates that the number of bosons in the Universe is between $10^{49}$ and $10^{51}$. One Friday, Milne was talking about Dirac's ideas on the importance of large numbers (not yet formalized) and it seemed natural to ask whether Eddington's number might be one of them. Milne's response was to demand an oral summary of Eddington's book the following Tuesday. Sadly, the only book from which a little general relativity might be gleaned that was borrowable over the weekend was Tolman's. The copy that has occupied shelfspace ever since is identical, but part of the 1966 reprinting.

For what it is worth, the book would not have been a bad choice. In the retrospect of a present need for a refresher course (unconnected with what follows), Tolman's interest in the relativistic definition of entropy must have been a great source of confusion all those years ago: it would have been irrelevant to Eddington's argument. But the essence of Einstein's general relativity is all there, if dryly. Beginners would have to work hard to get to the bottom of such things as the notion of the parallel displacement of vectors in curved space-time, for example.

But the real interest of Tolman's book lies in what it says, or rather does not say, about cosmology. First published in 1935, the text was probably evolved just a few years after Hubble's proof (or, rather, announcement, for some dispute the adequacy of the data) in 1930 that most other galaxies are receding from our own, and at a speed proportional to their distance.

More striking still, Tolman does not mention Aleksandr Friedmann, the Russian who in 1923 produced the solutions of Einstein's equations that are now the basis of most cosmological models; they languished in the Russian language until about the publication of Tolman's book, and are now known as the FriedmannRobertson-Walker solutions after their rediscoverers in the West. But Tolman has almost the next best thing, a discussion of the reasons why the Einstein static solution (with his cosmological constant included) must be thermodynamically unstable and why, for cosmologists, the choice must be between a continually expanding Universe and one that first expands and then contracts.

It is curious that the 1930s were almost dog-days for general relativity. It had been different a decade earlier. The appearance of Einstein's general relativity in 1915 stimulated a spate of interest. Minkowski produced a calculation of the gravitational field around a massive object, de Sitter a model for a Universe devoid of matter whose space-time nevertheless expanded inexorably. And there was Friedmann, working in obscurity.

It might have been thought that the demonstration that the Universe is indeed expanding would have given relativists a great fillip. The opposite seems to have been the case. People in the field were still struggling with the mathematical difficulties of curved space-time. On the other hand, empirically minded people took the line that general relativity would become a part of physics when there were meaningful ways in which it could be tested. They came along only in the 1960 s. Even now, while general relativity has survived all the tests that have been made on it, such tests as there are are local tests. We await the detection of gravitational waves from anywhere, not to mention from the distant quasars. General relativity remains untested on the scale of the Universe, the structure it was designed to describe.

Of necessity, Tolman does not tackle one of the great surprises in the mathematics of cosmology - the recognition that in the Universe as it is now, the evidence suggests that space-time is hardly curved at all in Einstein's sense, but almost flat. But that is why the newer textbooks are able to use Newtonian gravitation for describing the Universe in the large.

General relativity is indispensable only when it is necessary to calculate the properties of black holes and the like, where the gravitational fields are intense enough to point the glaring gap in the present understanding of gravitation in the Universe: the sheer absence of a bridge between general relativity in Einstein's classical sense and the quantum mechanics that has made rational the rest of physics. People have been beating their heads on that problem for a quarter of a century. If it is not soon cleared up, there seems every likelihood that general relativity will once more lapse into disuse, as in the 1930s.

Meanwhile, there is an important lesson to be learned from this tale: the old books - Tolman's is more a tract than a textbook - are more interesting than their almost forgotten familiarity suggests. They are important not just for what they say, but for what they are obliged by ignorance to omit to say. Perhaps one of the many public bodies that now exist to improve the public understanding of science should commission a study to chart the evolution of understanding from a close reading of textbooks picked up in secondhand bookshops.

John Maddox 